Grainger, C., Williams, D. M. \& Lind, S. E. (in press). Recognition memory and source memory in autism spectrum disorder: A study of the intention superiority and enactments effects. Autism: International Journal of Research and Practice,

\title{
Recognition memory and source memory in autism spectrum disorder: A study of the intention superiority and enactments effects.
}

\author{
Catherine Grainger ${ }^{\text {a,d }}$ \\ University of Kent
}

David M. Williams ${ }^{\mathrm{b}}$

University of Kent

Sophie E. Lind ${ }^{\mathrm{c}}$

City University London

This article may not exactly replicate the final version published in Autism: International Journal of Research and Practice. It is not a copy of the record. The article can be accessed at: https://uk.sagepub.com/en-gb/eur/journal/autism

${ }^{\text {a }}$ School of Psychology, Keynes College, University of Kent, Canterbury, Kent, CT2 7NP, United Kingdom. Email c.grainger@kent.ac.uk

${ }^{\mathrm{b}}$ School of Psychology, Keynes College, University of Kent, Canterbury, Kent, CT2 7NP, United Kingdom. Email d.m.williams@ @ent.ac.uk. Tel. +44 (0)1227 827652

${ }^{\mathrm{c}}$ Department of Psychology, City University London, Rhind Building, Northampton Square, London, EC1V 0HB, United Kingdom. Tel. +44 (0)20 7040 3372. Email sophie.lind.2@city.ac.uk

${ }^{d}$ Corresponding author: Catherine Grainger is now at the University of Stirling, 3B101, Cottrell Building, Stirling, FK9 4LA, UK. Phone: 01786-467670; Email:

catherine.grainger@stir.ac.uk 


\begin{abstract}
It is well established that neurotypical individuals generally show better memory for actions they have performed than actions they have observed others perform or merely read about, a so-called "enactment effect". Strikingly, research has also shown that neurotypical individuals demonstrate superior memory for actions they intend to perform in the future (but have not yet performed), an effect commonly known as the "intention superiority effect".

Although the enactment effect has been studied among people with ASD, the current study is the first to investigate the intention superiority effect in this disorder. This is surprising given the potential importance this issue has for general theory development, as well as for clinical practice. As such, this study aimed to assess the intention superiority and enactment effects in twenty-two children with ASD, and 20 IQ/age-matched neurotypical children. The results showed that children with ASD demonstrated not only undiminished enactment effects in recognition and source memory, but also (surprisingly for some theories) typical intention superiority effects. The implications of these results for theory, as well as clinical practice, are discussed.
\end{abstract}

Keywords: Autism spectrum disorder; Recognition memory; Source memory; Intention superiority effect; Enactment effect; Action monitoring; Episodic foresight; Motor encoding 
It is well established that neurotypical individuals show significantly better memory for actions that they have performed themselves, compared to actions that they have observed others perform, or actions they have merely read about (Baker-Ward, Hess, \& Flannagan, 1990; Engelkamp, 1998). Superior memory for actions one has performed is a robust effect within memory, and is typically known as the "enactment effect". Strikingly, however, studies have shown that it is not just memory for actions one has performed in the past that hold a privileged status in memory, but also memory for actions one intends to perform in the future (e.g., Goschke \& Kuhl, 1993; Marsh, Hicks, \& Bink, 1998). That is, individuals typically demonstrate better memory for actions when they make a plan to perform them at a future point than for actions they do not plan to perform. This "intention-superiority effect" appears to be consistent, as evidenced by superior recall (Jahn \& Engelkamp, 2003; Koriat, Ben-Zur, \& Nussbaum, 1990; Maylor, Chater, \& Brown, 2001), superior recognition (Jahn \& Engelkamp, 2003), faster recognition latencies (Goschke \& Kuhl, 1993), and faster lexical decision latencies (Marsh et al., 1998) for content associated with actions individuals intend to perform than for content associated with actions they do not intend to perform.

One possible explanation for the intention superiority effect is that, at the point of encoding one's intention, individuals imagine themselves actually carrying out that action at the appropriate future point. Engaging in such "episodic foresight" (or "episodic future thinking" / "prospection", as it is sometimes known) may result in particularly deep encoding of intended actions, which enhances subsequent memory for those actions relative to actions that one has not imagined performing. In line with this argument, it has been suggested that the chances of actually carrying out a previously formed intention increase when the context the intention is formed and encoded in is similar to the context it will be retrieved in (Gilbert, Armbruster, \& Panagiotidi, 2012). Studies have also shown that mentally imagining a visual image actives the neural areas associated with viewing that visual image (see e.g., Stokes et al., 2009). As such, when people form an intention to carry out a plan in the future (e.g., planning to turn off the bath taps before the water overflows), mentally imagining performing this intended action in the future (e.g., imagining yourself turning off the taps at the appropriate time) should increase the chances of the intention being carried out, as such mental simulation should increase the similarity between encoding and retrieval contexts. Following a similar argument, Brewer and Marsh (2010) have suggested that one's prospective memory ability (the ability to remember to carry out an intention at a future point) may be influenced by episodic foresight, and also suggest that engaging in episodic foresight processes during the encoding of an intention should strengthen the association 
between that intention and it's retrieval cues (thus making it more likely that a future intention will be carried out at the appropriate moment). Indeed studies have shown that an individual's episodic foresight ability significantly predicts one's prospective memory' ability (Nigro et al., 2014), and asking individuals to imagine a future event produces a significant improvement in their ability to carry out that event correctly in the future (Paraskevaides et al., 2010; see also Ford et al., 2012).

Although it is arguably intuitive to assume that episodic foresight underpins/contributes to the intention superiority effect, there is an alternative explanation of the effect that does not invoke such high-level mental simulation. Instead, some have suggested that it occurs as a result of the encoding of additional motor information associated with the to-be-performed action (Freeman \& Ellis, 2003; Koriat et al., 1990). Specifically, it has been suggested that when an individual encodes an intention to perform an action in the future, they activate motor information associated with performing that action. Thus, the planned actions are encoded in multiple formats, including an action-based format, which enhances subsequent memory for them. If this is true, then the intention superiority effect can be considered an extension of the enactment effect.

As discussed above, it is well established that neurotypical individuals show significantly better memory for actions that they have actually performed than actions that they have observed others perform, or actions they have merely read about (Baker-Ward et al., 1990; Engelkamp, 1998). This enactment effect is widely considered to result from additional motoric components involved in performing an action, leading to that action being more deeply encoded than actions than have been observed/read about (Engelkamp \& Zimmer, 1989). Along these lines, it has been suggested that the intention superiority effect could be considered an intention enactment effect, and superior memory for intended actions is purely the result of sensorimotor information encoded when an intention is formed (Freeman \& Ellis, 2003), which leads to that intention is encoded more deeply. Whether the future thinking theory or the motor theory provides a better explanation of the intention superiority effect is not yet clear, but either way the study of this effect has relevance for our understanding of autism spectrum disorder (ASD).

Autism spectrum disorder (ASD) is a developmental disorder diagnosed on the basis of social-communication deficits, and fixated interests and repetitive behaviours (see American Psychiatric Association, 2013). At the cognitive level, ASD is thought to be characterised by a particular profile of strengths and limitations in memory functioning (see Boucher \& Bowler, 2008) For example, on the one hand, there is robust evidence that 
individuals with ASD manifest impairments in both episodic memory, the ability to recall personally-experienced events, as well as episodic foresight, the ability to imagine events that are likely to be experienced in the future (Lind \& Bowler, 2010; Lind, Bowler, \& Raber, 2014; Lind, Williams, Bowler, \& Peel, 2014; Terrett et al., 2013). Recent evidence has also indicated a substantial deficit in prospective memory, at least when spontaneous (as opposed to prompted) retrieval of prior intentions is required for successful completion of a planned action (see e.g., Altgassen, Koban, \& Kliegel, 2012; Williams, Boucher, Lind, \& Jarrold, 2013; Williams, Jarrold, Grainger, \& Lind, 2014). On the other hand, there is highly robust evidence that individuals with ASD show a typical enactment effect in memory. In a review of 13 studies of the enactment effect in ASD, Grainger, Williams, and Lind (2014) reported that out of 21 analyses comparing memory for self-performed vs. observed actions in ASD, 17 did not find any between-group differences in the size of the enactment effect. On average, across studies involving a sum total of 239 individuals with ASD and 240 comparison participants, memory for self-performed actions was $10 \%$ better than memory for observed actions among individuals with ASD, and 11\% better among comparison participants (a negligible difference between groups; Cohen's $d=0.11$ ). This suggests strongly that individuals with ASD are able to monitor their own actions and that this monitoring enhances memory in a typical manner (see Williams, 2010). This pattern of impaired episodic memory in ASD, but intact action monitoring ability, supports the suggestion that individuals with ASD demonstrate selective impairments in self-awareness (see e.g., Williams, 2010), demonstrating typical physical self-awareness (e.g., actionmonitoring ability) but impairments in mental self-awareness (e.g., episodic memory) .

\section{The current study}

Despite the significant amount of research among people with ASD investigating the potential underpinnings of the intention superiority effect (i.e., future thinking, motor encoding), the intention superiority effect itself has - surprisingly - never been explored in this disorder. This is striking given the potential importance of this issue for general theory development, as well as for clinical practice (see p. 16 for a discussion). In the current study, the intention superiority effect, as well as the enactment effect, was explored among 22 children with ASD and 20 age-, IQ-, and sex-matched neurotypical comparison participants. During the experimental task, participants were presented with a series of written action phrases (e.g., "turn on the spot") during a study phase. On one third of trials, participants were asked to read the action statement aloud (Read condition). On another third of trials, 
they were asked to silently read and perform the action stated (Enact condition). Finally, in another third of trials still they were asked to silently read and were told that they would complete the action at the end of the task (Intend condition). Subsequently, participants' recognition and source memory for studied actions was assessed in a test phase.

Given the robust evidence that, among individuals with ASD, a) episodic foresight is impaired, and b) action monitoring is unimpaired, contrasting predictions can be made about the likelihood that the intention superiority effect will be diminished in ASD. On the one hand, if the intention superiority effect requires episodic future thinking, then the effect should be diminished in ASD. On the other hand, if the intention superiority effect results from additional encoding of motor information, then the intention superiority effect should be undiminished in ASD. The inclusion of an enactment condition (in which participants actually perform the written action phrases during the study phase) provides a further opportunity to confirm that action monitoring is unimpaired in ASD and, more importantly, to assess the relation between the intention superiority effect and the enactment effect in ASD.

\section{Method}

\section{Participants}

Twenty-two children with ASD and 20 neurotypical comparison children took part in this experiment, after they and their parents had given written, informed consent. Participants in the ASD group had formal diagnoses of Autistic Disorder or Asperger's disorder, according to established criteria (American Psychiatric Association, 2000; World Heath Organisation, 1993). To assess severity of ASD features, parents of participants with ASD completed the Social Responsiveness Scale (SRS; Constantino et al., 2003). In all but one case, participants with ASD scored above the defined cut-off for ASD on the SRS (total score $\geq 60$; Constantino et al., 2003). The remaining participant scored 55, which is just below the conventional ASD cut-off of 60. This participant had a formal diagnosis of Autistic Disorder.

Parents of neurotypical children also completed the SRS. All but one participant in the neurotypical group scored below the defined cut-off for ASD, with one participant scoring just above the cut off (66). After removing these participants from analyses, none of the results (or the study conclusions) changed substantively (i.e., no $p$ value changed from significant to non-significant or vice versa, and no effect size changed category - small, 
moderate, large), and thus these participants were included in analyses. Using the Wechsler Abbreviated Scale of Intelligence (WASI; Wechsler, 1999) the groups were equated closely for verbal IQ (VIQ), performance IQ (PIQ), and full-scale IQ (FSIQ). Both groups were also equated closely for chronological age. Participant characteristics are presented in Table 1.

\section{Materials and Procedures}

The Intention Superiority Task consisted of a study phase and a test phase. During the study phase participants were presented with 45 action phrases, each containing a key action word, 15 of which they read (Read condition), 15 of which they performed (Enact condition), and 15 of which they made the intention to perform at the end of the task (Intend condition). Three different 15-item lists of action phrases (e.g., "rub your stomach") were used as stimuli during the study phase of the task. A set of 45 novel action words was also compiled, which was used for the purpose of providing "lure" items during the test phase of the task. All four lists were equated for mean syllable length and mean spoken word frequency of action phrases, as indexed by Kucera and Francis (1967) and reported in the MRC Psycholinguistic Database (Coltheart, 1981). The adequacy of this matching was confirmed by a non-significant effect of List in a multivariate ANOVA (using Wilks' Lambda criterion) that included syllable length and word frequency as the dependent variables, $F(3,86)=.20, p=.894, \eta_{p}^{2}=.007$. During the study phase, each of the 15 item lists was assigned to a different condition (Read, Intend, Enact), and the order in which lists were assigned to each condition was counterbalanced across all participants. This created six different conditions of the task. During each condition, action phrases were presented to participants in a different, pseudo-randomised order, in which no more than two action phrases from any one condition appeared on successive trials.

Figure 1 shows a graphical representation of stimuli presentation on each trial of the task. During the study phase, action phrases were presented to participants on a computer screen which participants stood 1 metre away from. Participants were instructed that the beginning of each trial would be signalled by the presentation of one of three instructions (Read/Plan/Perform - corresponding to the conditions Read/Intend/Enact), which would appear individually at the top of the screen. Participants were told that after the instruction word had been presented, it would be followed by an action phrase, presented directly below the instruction. Participants were told that if the instruction "Read" appeared on the screen they should read the action phrase aloud. If the instruction "Perform" appeared on the screen participants were told that they should mime (act out) the action phrase. Finally, participants 
were instructed that if the instruction "Plan" appeared on the screen they should make a plan to perform the action at the end of the task. During each trial of the task the instruction word was presented individually on the screen for $1500 \mathrm{~ms}$, followed by the action phrase, which was presented below the instruction word for another $5000 \mathrm{~ms}$.

Before completing the study phase of the task, participants completed a practice task, which consisted of six trials (two from each condition). None of the action phrases that appeared in the practice task appeared during the experimental trials. After participants had completed the practice task they completed the experimental trials. During the experimental trials the experimenter observed the participant to check that they (a) performed the appropriate action on Enact trials of the task, (b) read aloud the action phrases on the Read trials, and (c) neither performed nor read the action phrases on Intend trials. If a participant did not perform an action during an Enact trial the experimenter took note of this trial and the trial was subsequently removed during data analysis. Similarly, if participants accidentally performed an action during a "Read" or "Intend" trial the experimenter took note of this, and this action phrase was also removed during data analysis. Errors in following the condition instructions correctly were very rare (and only occurred on three trials across two participants). Participants were informed, before starting the experimental trials, that after the study phase had been completed they would be asked some questions about what they had read, planned and performed, but were not explicitly told that their memory for the action phrases would be tested.

After the study phase, participants completed the test phase. Participants' recognition and source memory for the action phrases was tested during this phase. Participants were shown the set of action phrases individually on the computer screen and were asked to judge whether each one was "old" (had been presented to them previously during the study phase) or "new" (had not been presented during the study phase). If participants responded that an action phrase was old, they were asked to decide whether they thought the action was one that they had read, planned to perform, or actually performed during the task. The experimenter recorded participants' responses.

\section{Scoring}

Recognition memory performance. Recognition performance on the Intention Superiority Task was analysed using the measure of $d$-prime $\left(d^{\prime}\right)$, a parametric measure of item discrimination ${ }^{1}$. As a measure of recognition performance three separate $d$-prime scores 
were calculated using the formula below. In this equation $\mathrm{H}$ represents a hit rate (the proportion of old items participants correctly identified as "old") and FA represents false alarm rate (the proportion of new actions participants incorrectly identified as "old").

$$
d^{\prime}=z(H)-z(F A)
$$

Three separate $d^{\prime}$ scores were calculated, using separate hit rates based on the proportion of enacted actions correctly recognised, the proportion of intended actions recognised, and the proportion of read actions recognised. A single false alarm rate was used to calculate all three $d^{\prime}$ scores, since false alarm rates were derived from performance on distractor items, which by definition were neither read, intended nor enacted during the task.

Source monitoring performance. Source monitoring performance on the experimental task was assessed as hit rates (the proportion of items participants made the correct source judgement for, for example the proportion of enacted items participants correctly identified as items they had performed). Three separate hit rates were calculated, representing the proportion of enacted actions participants made correct source monitoring judgements for, the proportion of intended actions participants made correct source monitoring judgements for, and the proportion of read actions participants made correct source monitoring judgements for.

Alpha levels and effect sizes. A standard alpha level of .05 was used to determine statistical significance and reported significance values are for two-tailed tests. Where ANOVAs were used, $\eta_{p}^{2}$ values are reported as measures of effect size $(\geq .01=$ small effect, $\geq$ $.06=$ moderate effect, $\geq .14$ = large effect; Cohen, 1969). Where $t$-tests were used, Cohen's $d$ values are reported as measures of effect size $(\geq .0 .20=$ small effect, $\geq 0.50=$ moderate effect; $\geq 0.80=$ large effect; Cohen, 1969). When correlations were used $\geq .30$ was considered a small effect, $\geq .50$ was considered a moderate effect and $\geq .70$ was considered a large effect (Cohen, 1969).

\section{Results}

\section{Intention Superiority Task}

Performance in each condition of the intention superiority task in the ASD and neurotypical group can be seen in Table 2. Before analysing group differences in performance, a series of one-sample $t$-tests was carried out to establish whether performance on the intention superiority task was at floor or ceiling level, for any of the memory measures. These $t$-tests indicated that, in both the ASD and neurotypical groups, the 
proportion of actions correctly recognised in each condition significantly differed from floor or ceiling level performance, all $t \mathrm{~s} \geq 3.47$, all $p \mathrm{~s} \leq .003$, as did the proportion of actions participants made correct source monitoring judgements for, all $t \mathrm{~s} \geq 4.55$, all $p \mathrm{~s}<.001$.

Recognition memory. D-prime scores for recognition performance in each condition of the task (Read/Intend/Enact) are reported in Table 2, for both the ASD and neurotypical group. A 2 (Group: ASD/neurotypical) $\times 3$ (Condition: Read/Intend/Enact) mixed ANOVA was conducted on these data. A significant main effect of Condition was found, $F(2,80)$ $=118.50, p<.001, \eta_{p}^{2}=.75$. This reflected the fact that across both groups, recognition memory was significantly greater for actions participants had enacted than for actions participants had intended, and recognition memory for actions participants had intended was significantly greater than actions participants had read, all $t s \geq 3.13$, all $p \mathrm{~s} \leq .003$, and all $d \mathrm{~s} \geq$ 0.42 (see Figure 2 for a graphical representation of these results).

However, there was no significant main effect of Group, $F(1,40)=2.09, p=.156, \eta_{p=}^{2}$ .05 , and - more importantly - no significant interaction between Group and Condition $F(2$, $60)=0.38, p=.685, \eta_{p}^{2}=.01$. These results indicate that the ASD group demonstrated a similar pattern of recognition memory to neurotypical children on the task (demonstrating typical enactment effects and typical intention superiority effects).

Source Monitoring. Hit rates for source monitoring performance in each condition of the task (Read/Intend/Enact) are also reported for both the ASD and neurotypical groups in Table 2. A 2 (Group: ASD/neurotypical) $\times 3$ (Condition: Read/Intend/Enact) mixed ANOVA was conducted on these data. A significant main effect of Condition was found, $F(2,80)=$ $133.58, p<.001, \eta_{p}^{2}=.770$. This reflected the fact that participants, across both groups, demonstrated significantly better source monitoring performance for actions they had enacted than for actions they had intended to perform, and significantly greater source monitoring performance for actions participants had intended to perform, than actions they had read, all $t s \geq 7.75$, all $p s<.001$, and all $d s \geq 1.57$ (see Figure 3 for a graphical representation of these results).

However, again there was no significant main effect of Group, $F(1,40)=0.09, p=$ $.768, \eta_{p=}^{2} .002$, and no significant interaction between Group and Condition $F(2,80)=0.24$, $p=.788, \eta_{p}^{2}<.01$. As such, there were no significant differences between the groups in either overall levels or patterns of performance across conditions.

To summarise, on both tests of memory (recognition and source monitoring) participants in both the ASD and neurotypical groups showed better memory for actions that 
they had enacted than actions they had read or actions they intended to perform (thus demonstrating clear enactment effects). Additionally, on both tests of memory participants in both groups showed better memory for actions that they had intended to perform than actions they had simply read (demonstrating intention superiority effects). This pattern of memory performance did not differ between ASD participants and neurotypical participants on any measure, as indicated by no significant interactions between participants' diagnostic group and their memory for read/intended/enacted actions for either recognition or source memory performance.

\section{Associations between the enactment effect and the intention-superiority effect}

In order to establish the extent to which the enactment effect (i.e., the memory gain from performing actions) was associated with the intention superiority effect (i.e., the memory gain from intending to perform actions), a series of partial correlations was run. Among each participant group, the association between recognition memory performance in the Enact condition and recognition memory performance in the Intend condition, controlling for recognition memory performance in the Read condition, was computed. Likewise, the association between source memory performance in the Enact condition and source memory performance in the Intend condition, controlling for source memory performance in the Read condition, was computed. Partialling out performance in the Read condition allows us to control for "general memory" ability in each of these analyses. As such, gain an indication of whether the additional recognition/source monitoring benefit of enactment and the additional recognition/source monitoring benefit of intending to perform an action are related.

For the ASD group, there was a significant association between memory for intended and enacted actions (when controlling for memory for read action), in both recognition memory, $r=.64, p=.002$, and source memory, $r=.46, p=.034$. These results indicate that the larger the enactment effect in children with ASD, the larger the intention-superiority effect, in both recognition and source memory. In the neurotypical group there was also a significant correlation between memory for intended and enacted actions in recognition memory, $r=.50, p=.029$. However, there was no significant correlation in source memory between memory for intended and performed action, $r=.15, p=.546$. Fisher's $z$ tests indicated that there were no significant differences between the ASD and neurotypical groups in the size of these correlations, all $z \mathrm{~s} \leq 1.04$, all $p \mathrm{~s} \geq .30$.

\section{Discussion}


Until now, no study has explored the intention superiority effect in ASD. As such, the primary aims of this study were to explore the extent to which individuals with ASD demonstrate a typical intention superiority effect, as well as to replicate the established finding of undiminished enactment effect among people with this disorder. The central results from the study found no indication that children with ASD manifest diminished intention superiority or enactment effects in either recognition or source memory. In each ANOVA, the critical Group (ASD/comparison) x Condition (Read/Intend/Enact) interaction effects were non-significant and small in magnitude. This suggests that participants with ASD and comparison participants showed similar levels and patterns of performance on the experimental task; both groups showed an identical pattern of performance, recognising and monitoring the source of action terms that were enacted better than action terms that were intended to be enacted. In turn, both enacted and planned actions were recognised and source monitored better than action terms that were merely read (hence, enacted actions > intended actions > read actions).

It is interesting that the intention-superiority effect demonstrated by individuals with ASD was found in both source memory and recognition memory. Although recognition memory may potentially be underpinned by the semantic memory system (familiarity), source memory (which involves identifying something about the context under which a memory was acquired) is generally considered to be underpinned by the episodic system (recollection), because only episodic retrieval involves identifying the spatio-temporal context of the recollected episode (Johnson, Hashtroudi, \& Lindsay, 1993; Wheeler et al., 1997). Given adults with ASD demonstrated typical intention-superiority effects on measures of both source memory and recognition memory, this suggests that the ISE observed in the ASD group was not specific to familiarity-based memory processes. However, although the test procedure used in the reported study tested memory using both familiarity and recollection-based measures, it was the case that memory performance on both measures was cued. As such, future research is needed to test whether the intention-superiority effect is typical during spontaneous retrieval of ones intentions.

The finding that individuals with ASD also showed a typical enactment effect in recognition and source memory provides yet further evidence that action monitoring abilities (and, hence, a sense of agency) are undiminished and enhance memory for completed actions in this disorder (see e.g., Grainger et al., 2014). These results are in keeping with a large body of studies demonstrating undiminished enactment effects in ASD (Grainger et al., 2014; e.g., Hare, Mellor, \& Azmi, 2007; Lind \& Bowler, 2009; Maras, Memon, Lambrechts, \& 
Bowler, 2012; Summers \& Craik, 1994; Williams \& Happé, 2009), as well as with other studies of action monitoring in ASD (Blakemore et al., 2006; e.g., David et al., 2008; but see Zalla \& Sperduti, 2015 for an alternative perspective). Moreover, these results provide the first evidence about the ways in which planning to carry out actions in the future influences subsequent retrospective memory for those actions in ASD. The finding of an undiminished (indeed, slightly enhanced) intention superiority effect in ASD suggest that forming the intention to perform an action in the future results in subsequently superior memory for that action among individuals with this disorder.

From a clinical perspective, finding that individuals with ASD demonstrate typical intention superiority has important implications. Specifically, typical intention superiority in ASD could potentially be used as a method to support learning, particularly within the school environment. In much the same way as the enactment effect and "subject performed tasks" have been utilised in educational contexts, and been shown to be beneficial to learning in children with ASD (Summers \& Craik, 1994), so too could the intention superiority effect. This is particularly important when it is not always practical to physically enact a task. In such cases asking children instead to plan to perform a task later (at the appropriate time) could prove to be a useful learning aid. Learning techniques that utilise intention superiority in ASD may also be beneficial for supporting social interactions, helping individuals to remember social rules and hence reduce the amount of "online" processing needed during interactions. For example, asking children with ASD to plan performing specific strategies during social interactions (e.g., asking children to plan making eye contact with an individual when talking to them) may also prove a useful learning aid.

Although the focus of the current study was on further understanding the nature of memory processes in ASD, the results may also be relevant to theories of the underpinnings of the intention superiority effect. Individuals in the both the ASD and neurotypical group demonstrated typical enactment effects and typical intention superiority effects. Furthermore, in three out of four partial correlation analyses (across both ASD and comparison participants) the association between the intention superiority effect and the enactment effect was significant. These results support the suggestion that the intention superiority effect and enactment effect rely on the same underlying processes, presumably motor encoding (see Freeman \& Ellis, 2003 for further evidence in favour of the motor encoding theory).

Whether "action superiority" can adequately and entirely account for the intention superiority effect is not yet certain. It has been argued that often the specific motor components associated with a future intention are not known to an individual when they 
encode the intention, and some intentions may be too rich/complex to be represented through sensorimotor encoding (McDaniel \& Einstein, 2007). McDaniel and Einstein (2007) question whether individuals engage in sensorimotor encoding of complex intention such as the intention to take a trip (which itself encompasses the intention to perform several actions e.g., to pack your bag, check in to your flight, cancel your mail etc.).

The underlying processes involved in intention-superiority are not yet certain. However this study has demonstrated that individuals with ASD demonstrate typical intention-superiority, in both recognition memory and source monitoring memory. These findings represent a relative memory strength in ASD, and should thus inform intervention efforts aimed at remediating specific memory impairments in this disorder. 


\section{Footnotes}

${ }^{1}$ It should be highlighted that when corrected hit rates (a non-parametric measure of recognition ability) are used to calculate recognition performance (instead of $d$ '), none of the results (or the study conclusions) change substantively (i.e., no $p$ value changed from significant to non-significant or vice versa, and no effect size changed category - small, moderate, large). 
Tables

Table 1: Means (SDs) and inferential statistics for participant characteristics.

\begin{tabular}{lcccccc}
\hline & \multicolumn{2}{c}{ Group } & & & \\
\cline { 2 - 3 } & ASD & Neurotypical & $t$ & $p$ & Cohen's \\
& $(n=22 ; 19$ male $)$ & $(n=20 ; 20$ male $)$ & & & $d$ \\
\hline Age (years) & $13.42(1.12)$ & $13.22(1.01)$ & 0.62 & .539 & 0.19 \\
VIQ & $106.00(19.34)$ & $106.90(14.43)$ & 0.21 & .838 & 0.05 \\
PIQ & $106.05(12.90)$ & $109.80(14.48)$ & 0.89 & .379 & 0.27 \\
FSIQ & $106.73(11.84)$ & $109.50(15.00)$ & 0.67 & .508 & 0.20 \\
SRS Total & $83.59(9.87)$ & $43.25(7.86)$ & 14.45 & $<.001$ & 4.52 \\
\hline
\end{tabular}

SRS: Social Responsiveness Scale (Constantino et al., 2003); VIQ = verbal IQ; PIQ = performance IQ; FSIQ = full scale IQ. 
Table 2: Means (SDs) and inferential statistics for group differences in performance on the intention superiority task.

\begin{tabular}{lcccccc}
\hline Memory Type & Condition & \multicolumn{2}{c}{ Group } & & \\
\cline { 3 - 7 } & & ASD & Neurotypical & $t$ & $p$ & Cohen's \\
& & $(n=22)$ & $(n=20)$ & & & $D$ \\
\hline Recognition (d') & Read & $1.75(0.73)$ & $2.02(0.65)$ & 1.25 & .220 & 0.39 \\
& Intend & $2.06(0.60)$ & $2.26(0.65)$ & 1.04 & .304 & 0.32 \\
& Enact & $3.03(0.83)$ & $3.38(0.74)$ & 1.48 & .147 & 0.45 \\
Source & Read & $.35(.19)$ & $.36(23)$ & .192 & .848 & 0.04 \\
Monitoring & Intend & $.67(.16)$ & $.63(.19)$ & .615 & .542 & 0.23 \\
(Hit Rate) & Enact & $.90(.08)$ & $.89(.12)$ & .355 & .724 & 0.10 \\
& & & & & & \\
\hline
\end{tabular}


Figures

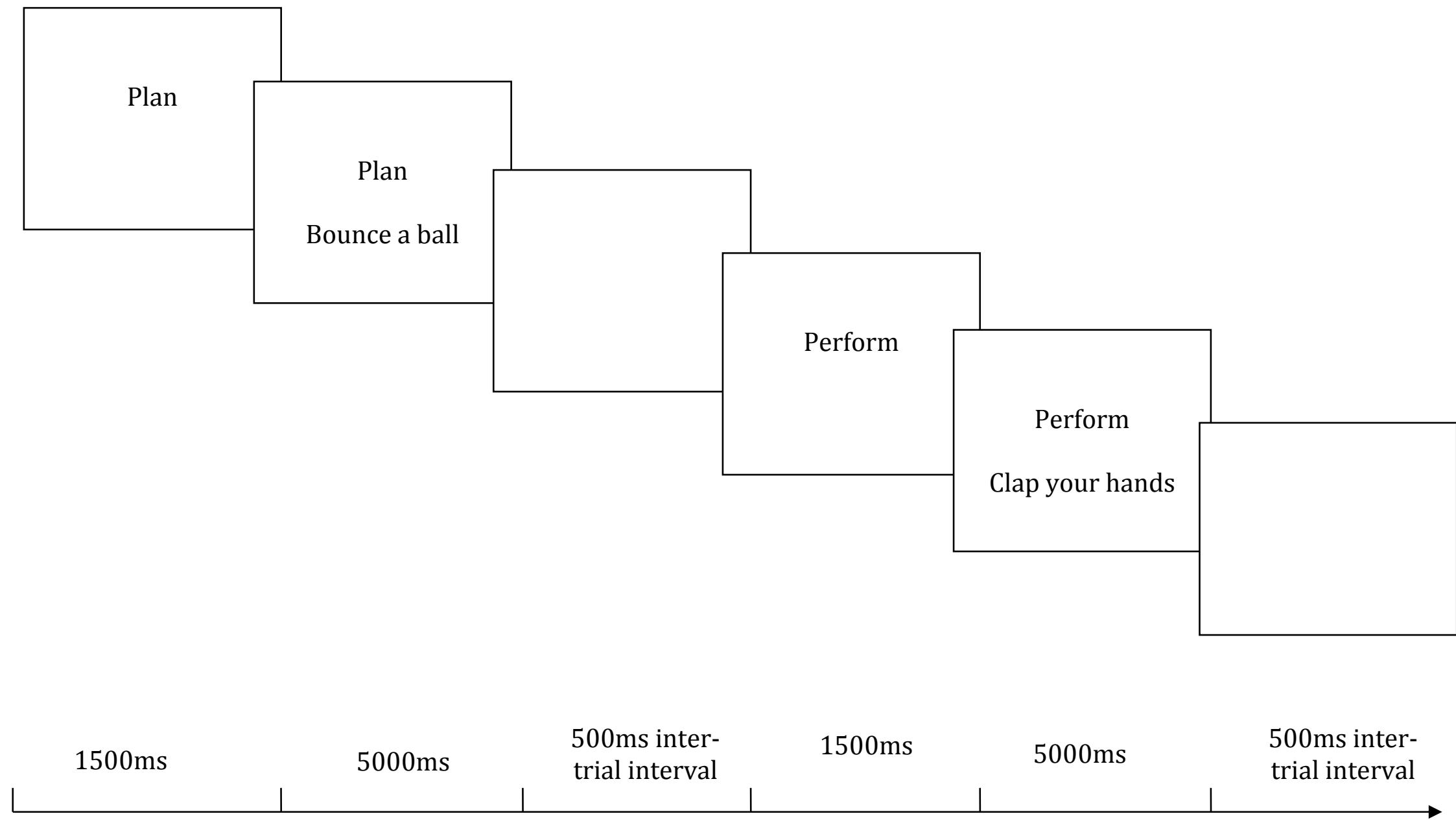

Figure 1: Graphical representation of the procedure used during the study phase of the Intention Superiority Task

(providing an example of two trials). 


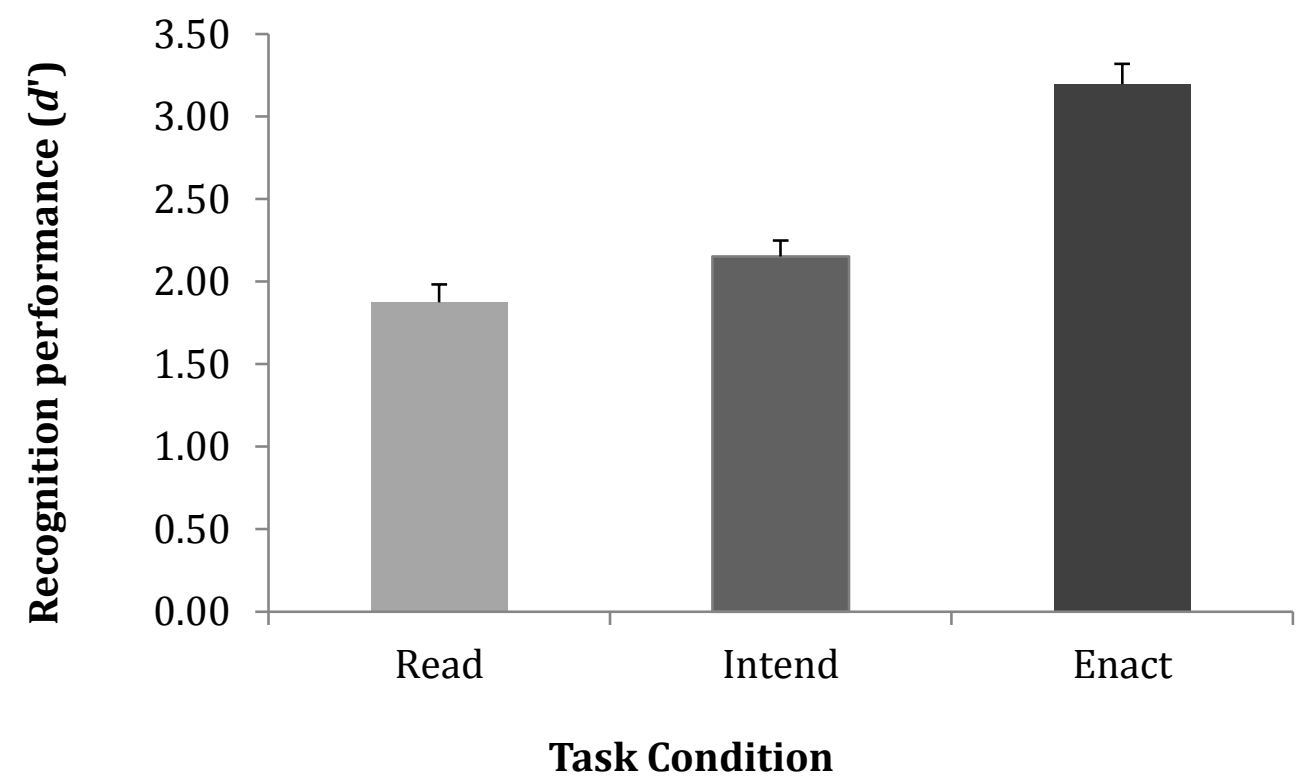

Figure 2: Recognition performance (d'scores) for combined (neurotypical and ASD) groups for performance in each condition of the task. Error bars represent standard error of the mean. 


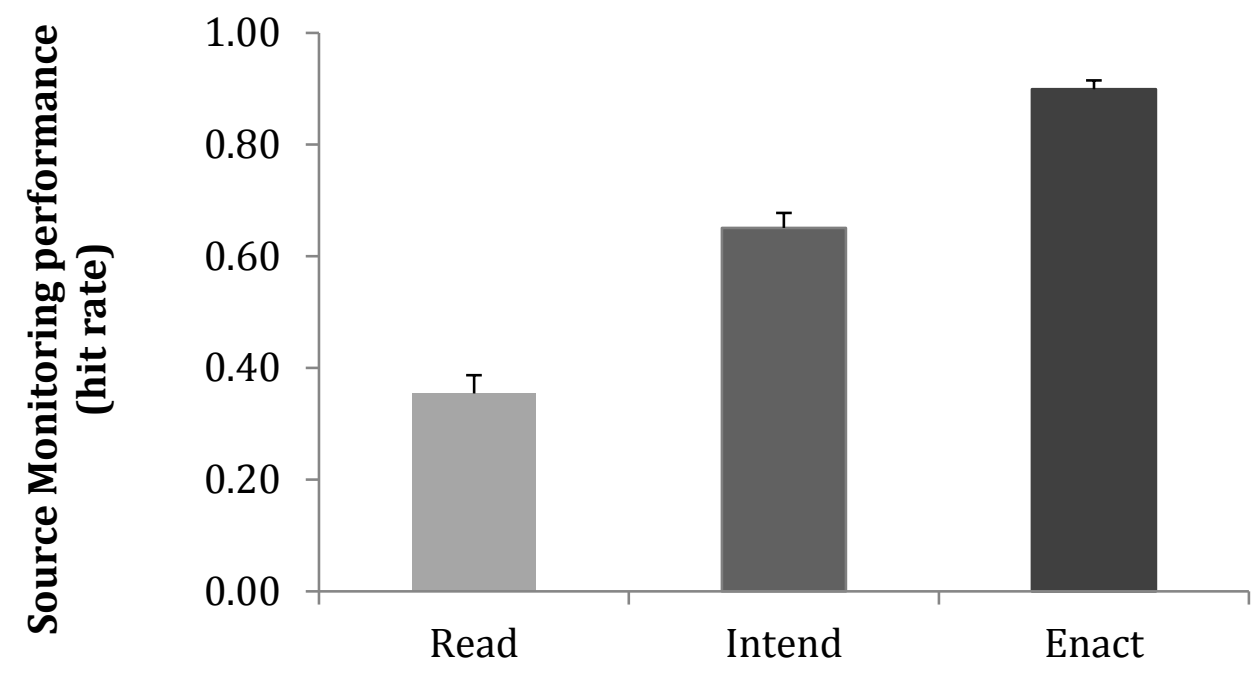

\section{Task Condition}

Figure 3: Source monitoring performance (hit rate) for combined (neurotypical and ASD) groups for performance in each condition of the task. Error bars represent standard error of the mean. 


\section{References}

Altgassen, M., Koban, N., \& Kliegel, M. (2012). Do adults with autism spectrum disorders compensate in naturalistic prospective memory tasks? Journal of Autism and Developmental Disorders, 42(10), 2141-2151. doi:10.1007/s10803-012-1466-3.

American Psychiatric Association. (2000). Diagnostic and statistical manual of mental disorders (4th edition, text revised) (DSM-IV-TR). Washington DC: American Psychiatric Association.

American Psychiatric Association. (2013). Diagnostic and statistical manual of mental disorders (5th edition). Washington DC: American Psychiatric Association.

Baker-Ward, L., Hess, T. M., \& Flannagan, D. A. (1990). The effects of involvement on children's memory for events. Cognitive Development, 5(1), 55-69. doi:10.1016/08852014(90)90012-I.

Blakemore, S. J., Tavassoli, T., Calo, S., Thomas, R. M., Catmur, C., Frith, U., \& Haggard, P. (2006). Tactile sensitivity in Asperger syndrome. Brain Cognition, 61(1), 5-13. doi:10.1016/j.bandc.2005.12.013.

Boucher, J., \& Bowler, D. M. (2008). Memory in Autism. Cambridge: Cambridge University Press.

Brewer, G. A., \& Marsh, R. L. (2010). On the role of episodic future simulation in encoding of prospective memories. Cognitive Neuroscience, 1, 81-88. doi: $10.1080 / 17588920903373960$

Cohen, J. (1969). Statistical Power Analysis for the Behavioural Sciences. San Diego, CA: Academic Press.

Coltheart, M. (1981). The MRC psycholinguistic database. . Quarterly Journal of Experimental Psychology Section A: Human Experimental Psychology, 33(33), 497505. doi:10.1080/14640748108400805.

Constantino, J. N., Davis, S. A., Todd, R. D., Schindler, M. K., Gross, M. M., Brophy, S. L., . . Reich, W. (2003). Validation of a brief quantitative measure of autistic traits: Comparison of the social responsiveness scale with the autism diagnostic interviewrevised. Journal of Autism and Developmental Disorders, 33(4), 427-433. doi:10.1023/A:1025014929212 .

David, N., Gawronski, A., Santos, N. S., Huff, W. G., Lehnhardt, F. G., Newen, A., \& Vogeley, K. (2008). Dissociation between key processes of social cognition in autism: 
Impaired mentalizing but intact sense of agency. Journal of Autism and Developmental Disorders, 38(4), 593-605. doi:10.1007/s10803-007-0425-х.

Engelkamp, J. (1998). Memory for Actions. Hove: Psychology Press.

Engelkamp, J., \& Zimmer, H. D. (1989). Memory for action events - A new field of research. Psychological Research-Psychologische Forschung, 51(4), 153-157. doi:10.1007/BF00309142.

Ford, R. M., Driscoll, T., Shum, D., \& Macaulay, C. E. (2012). Executive and theory-of-mind contributions to event-based prospective memory in children: Exploring the selfprojection hypothesis. Journal of Experimental Child Psychology, 111(3), 468-489. doi: 10.1016/j.jecp.2011.10.006

Freeman, J. E., \& Ellis, J. A. (2003). The representation of delayed intentions: A prospective subject-performed task? Journal of Experimental Psychology-Learning Memory and Cognition, 29(5), 976-992. doi:10.1037/0278-7393.29.5.976.

Gilbert, S. J., Armbruster, D. J., \& Panagiotidi, M. (2012). Similarity between brain activity at encoding and retrieval predicts successful realization of delayed intentions. Journal of Cognitive Neuroscience, 24(1), 93-105.doi:10.1162/jocn_a_00094

Goschke, T., \& Kuhl, J. (1993). Representation of intentions: Persisting activation in memory. Journal of Experimental Psychology: Learning, Memory, and Cognition, 19(5), 1211. doi:10.1037/0278-7393.19.5.1211.

Grainger, C., Williams, D. M., \& Lind, S. (2014). Online Action Monitoring and Memory for Self-Performed Actions in Autism Spectrum Disorder. Journal of Autism and Developmental Disorders, 1-14. doi:10.1007/s10803-013-1987-4.

Hare, D. J., Mellor, C., \& Azmi, S. (2007). Episodic memory in adults with autistic spectrum disorders: Recall for self- versus other-experienced events. Research in Developmental Disabilities, 28(3), 317-329. doi:10.1016/j.ridd.2006.03.003.

Jahn, P., \& Engelkamp, J. (2003). Design-effects in prospective and retrospective memory for actions. Experimental Psychology, 50(1), 4-15. doi:10.1021//1618-3169.50.1.4.

Johnson, M. K., Hashtoudi, S., \& Lindsay, D. S. (1993). Source monitoring. Psychological Bulletin, 114, 3-28. doi: 10.1037/0033-2909.114.1.3.

Koriat, A., Ben-Zur, H., \& Nussbaum, A. (1990). Encoding information for future action: memory for to-be-performed tasks versus memory for to-be-recalled tasks. Memor and Cognition, 18(6), 568-578. doi:10.3758/BF03197099.

Kucera, H., \& Francis, W. N. (1967). Computational analysis of present day American English. Providence, RI: Brown University Press. 
Lind, S. E., \& Bowler, D. M. (2009). Recognition memory, self-other source memory, and theory-of-mind in children with autism spectrum disorder. Journal of Autism and Developmental Disorders, 39(9), 1231-1239. doi:10.1007/s10803-009-0735-2.

Lind, S. E., \& Bowler, D. M. (2010). Episodic memory and episodic future thinking in adults with autism. Journal of Abnormal Psychology, 119(4), 896-905. doi:10.1037/a0020631.

Lind, S. E., Bowler, D. M., \& Raber, J. (2014). Spatial navigation, episodic memory, episodic future thinking, and theory of mind in children with autism spectrum disorder: Evidence for impairments in mental simulation? Frontiers in Psychology, 5. doi:10.3389/fpsyg.2014.01411.

Lind, S. E., Williams, D. M., Bowler, D. M., \& Peel, A. (2014). Episodic Memory and Episodic Future Thinking Impairments in High-Functioning Autism Spectrum Disorder: An Underlying Difficulty With Scene Construction or Self-Projection. Neuropsychology, 28(1), 55-67. doi:10.1037/neu0000005.

Maras, K., Memon, A., Lambrechts, A., \& Bowler, D. (2012). Recall of a live and personally experienced eyewitness event by adults with autism spectrum disorder. Journal of Autism and Developmental Disorders, 43(8), 1-13. doi:10.1007/s10803-012-1729-z.

Marsh, R. L., Hicks, J. L., \& Bink, M. L. (1998). Activation of completed, uncompleted, and partially completed intentions. Journal of Experimental Psychology-Learning Memory and Cognition, 24(2), 350-361. doi:10.1037//0278-7393.24.2.350.

Nigro, G. Brandimonte, M. Cicogna, P. \& Cosenza, M. (2014). Episodic future thinking as a predictor of children's prospective memory. Journal of Experimental Child Psychology, 127, 82-94. doi: 10.1016/j.jecp.2013.10.013

Maylor, E. A., Chater, N., \& Brown, G. D. A. (2001). Scale invariance in the retrieval of retrospective and prospective memories. Psychonomic Bulletin \& Review, 8(1), 162167. doi:10.3758/bf03196153.

McDaniel, M. A., \& Einstein, G. O. (2007). Prospective memory: An overview and synthesis of an emerging field: Sage Publications.

Paraskevaides, T., Morgan, C. J. A., Leitz, J. R., Bisby, J. A., Rendell, P. G., \& Curran, H. V. (2010). Drinking and future thinking: Acute effects of alcohol on prospective memory and future simulation. Psychopharmacology, 208, 301-308. doi: 10.1007/s00213-009$1731-0$ 
Stokes, M., Thompson, R., Cusack, R., \& Duncan, J. (2009). Top-down activation of shapespecific population codes in visual cortex during mental imagery. The Journal of Neuroscience, 29(5), 1565-1572. doi:10.1523/JNEUROSCI.4657-08.2009.

Summers, J. A., \& Craik, F. I. M. (1994). The effects of subject-performed tasks on the memory performance of verbal autistic-children. Journal of Autism and Developmental Disorders, 24(6), 773-783. doi:10.1007/BF02172285.

Terrett, G., Rendell, P. G., Raponi-Saunders, S., Henry, J. D., Bailey, P. E., \& Altgassen, M. (2013). Episodic future thinking in children with autism spectrum disorder. Journal of Autism and Developmental Disorders,, 43(11), 2558-2568. doi:10.1007/s10803-0131806-y.

Wechsler, D. (1999). Wechsler abbreviated scale of intelligence. New York, NY: The Psychological Corporation: Harcourt Brace \& Company.

Wheeler, M., Stuss, D., \& Tulving, E. (1997). Towards a theory of episodic memory: The frontal lobes and autonoetic consciousness. Psychological Bulletin, 121, 331-354.

Williams, D. M., Boucher, J., Lind, S., \& Jarrold, C. (2013). Time-based and event-based prospective memory in autism spectrum disorder: the roles of executive function and theory of mind, and time-estimation. Journal of Autism and Developmental Disorders, 43(7), 1555-1567.

Williams, D. M. (2010). Theory of own mind in autism. Autism, 14(5), 474-494. doi:10.1177/1362361310366314.

Williams, D. M., \& Happé, F. (2009). Pre-conceptual aspects of self-awareness in autism spectrum disorder: The case of action-monitoring. Journal of Autism and Developmental Disorders, 39(2), 251-259. doi:10.1007/s10803-008-0619-х

Williams, D. M., Jarrold, C., Grainger, C., \& Lind, S. E. (2014). Diminished time-based, but undiminished event-based, prospective memory among intellectually high-functioning adults with autism spectrum disorder: relation to working memory ability. Neuropsychology, 28(1), 30.

World Heath Organisation. (1993). International classification of mental and behavioural disorders: Clinical descriptions and diagnostic guidelines (10th edn.) Geneva, Switzerland: World Heath Organisation.

Zalla, T., \& Sperduti, M. (2015). The Sense of Agency in Autism Spectrum Disorders: a Dissociation between Prospective and Retrospective Mechanisms? Frontiers in Psychology, 6. doi:10.3389/fpsyg.2015.01278. 